\title{
Erratum to: A phylogenomic reappraisal of family-level divisions within the class Halobacteria: proposal to divide the order Halobacteriales into the families Halobacteriaceae, Haloarculaceae fam. nov., and Halococcaceae fam. nov., and the order Haloferacales into the families, Haloferacaceae and Halorubraceae fam nov.
}

\author{
Radhey S. Gupta - Sohail Naushad - Reena Fabros • Mobolaji Adeolu \\ Published online: 17 September 2016 \\ (C) Springer International Publishing Switzerland 2016
}

Erratum to: Antonie van Leeuwenhoek (2016)

109:565-587

DOI 10.1007/s10482-016-0660-2

On page 583 and in Fig. 7 of this paper the genus Halogranum, in addition to its correct assignment to the family Haloferacaceae, has also been erroneously assigned to the family Halorubraceae. Additionally, on page 582 and in Fig. 7 of this paper, the genus Natronomonas was erroneously placed within the family Halobacteriaceae instead of its correct placement in the family Haloarculaceae. It is emphasised here that the genus Halogranum belongs to the family Haloferacaceae whereas the genus Natronomonas belongs to the family Haloarculaceae. In Fig. 7, and in the description of the family Haloarculaceae, the correct numbers of CSIs and CSPs specific for this family are 10 and 20, respectively, instead of 11 and 19 as originally indicated; this error is also now corrected. The corrected version of Fig. 7 is reprinted here and the correct descriptions of the families Halobacteriaceae, Haloarculaceae, Haloferacaceae and Halorubraceae from pages 582-583 of the published article should read as follows:

The online version of the original article can be found under doi:10.1007/s10482-016-0660-2.

R. S. Gupta $(\bowtie) \cdot$ S. Naushad · R. Fabros · M. Adeolu Department of Biochemistry and Biomedical Sciences, McMaster University, Hamilton, ON L8N 3Z5, Canada e-mail: gupta@mcmaster.ca
Emended description of the family

Halobacteriaceae Gibbons 1974

(approved Lists 1980)

The family Halobacteriaceae contains the type genus Halobacterium (Oren et al. 2009) and the genera Haladaptatus (Cui et al. 2010d), Halalkalicoccus (Xue et al. 2005), Halarchaeum (Minegishi et al. 2010a), Haloarchaeobius (Yuan et al. 2015), Halomarina (Inoue et al. 2011), Halorubellus (Cui et al. 2012), Halorussus (Cui et al. 2010c), Halovenus (Makhdoumi-Kakhki et al. 2012), Natronoarchaeum (Shimane et al. 2010), and Salarchaeum (Shimane et al. 2011). The description of the family is the same as that of the order Halobacteriales given by Gupta et al. (2015) with the following modifications: The members of this order also lack the CSIs and CSPs that are specific for the families Haloarculaceae and Halococcaceae.

\section{Description of Haloarculaceae fam. nov.}

Haloarculaceae (Ha.lo.ar.cu.la.ce'ae. N.L. fem. n. Haloarcula type genus of the family; -aceae ending to denote a family; N.L. fem. pl. n. Haloarculaceae the family whose nomenclatural type is the genus Haloarcula).

The family Haloarculaceae contains the type genus Haloarcula (Oren et al. 2009) and the genera Halapricum (Song et al. 2014), Halomicroarcula 


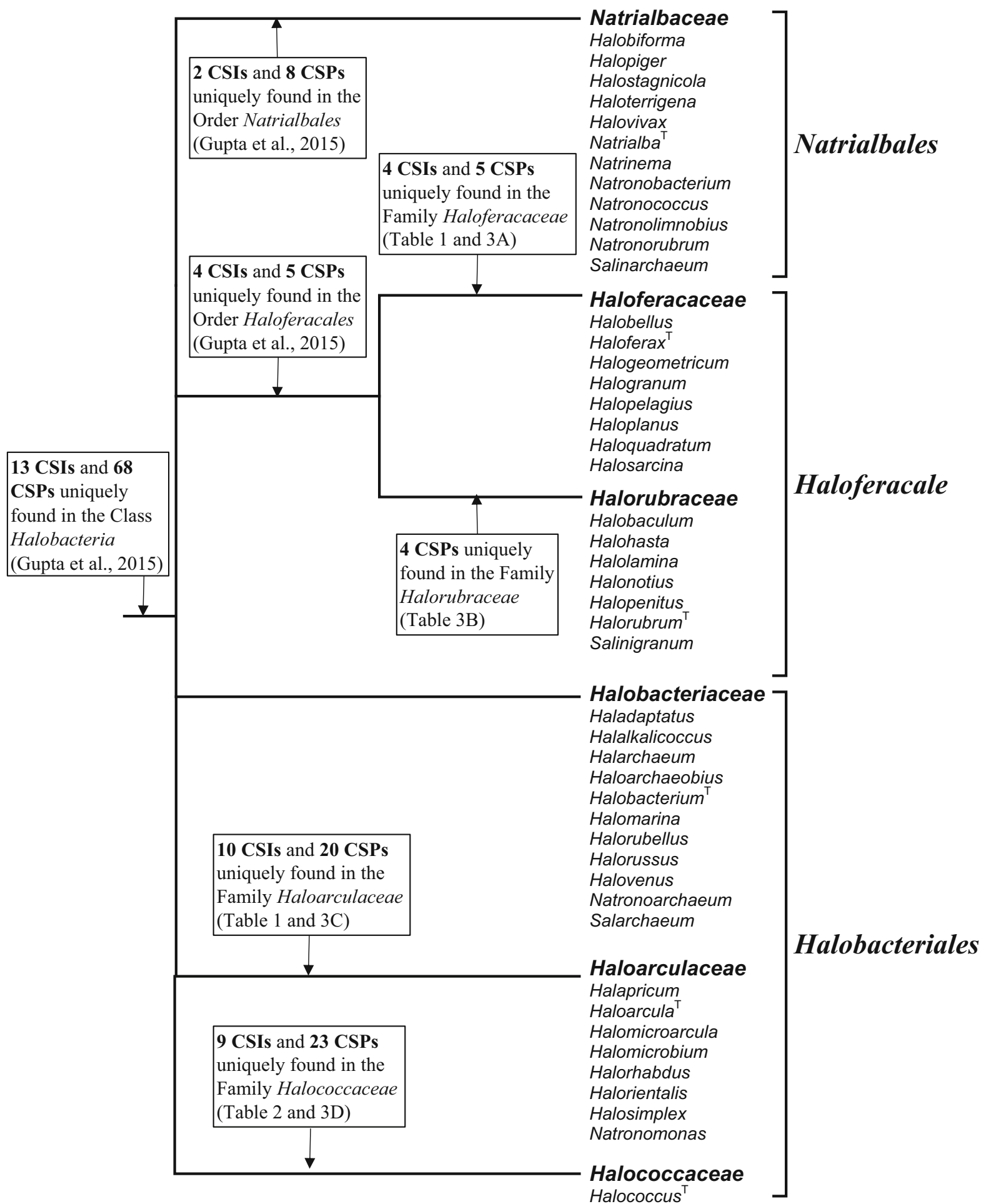

Fig. 7 A summary diagram depicting the distribution of identified CSIs and CSPs within the class Halobacteria and the proposed families described in this study. The genera within each family are listed under the corresponding family name. The superscript letter $\mathrm{T}$ beside a genus indicates that it is the type genus of the family 
(Echigo et al. 2013), Halomicrobium (Oren et al. 2002), Halorhabdus (Antunes et al. 2008), Halorientalis (Amoozegar et al. 2014), Halosimplex (Han and Cui 2014), and Natronomonas (Burns et al. 2010b). The description of the family is the same as that of the order Halobacteriales given by Gupta et al. (2015) with the following modifications: the members of the family Haloarculaceae can be distinguished from the Halobacteriaceae and all other archaea by the ten CSIs listed in Table 1 and by the twenty CSPs listed in Table 3C.

\section{Emended description of the family Haloferacaceae Gupta 2015}

The family Haloferacaceae contains the type genus Haloferax (Oren et al. 2009) and the genera Halobellus (Cui et al. 2011b), Halogeometricum (Cui et al. 2010e), Halogranum (Cui et al. 2010b, 2011c), Halopelagius (Zhang et al. 2013), Haloplanus (Qiu et al. 2013), Haloquadratum (Burns et al. 2007), and Halosarcina (Cui et al. 2010a). The description of the family is the same as that of the order Haloferacales given by Gupta et al. (2015) with the following modifications: the members of the family Haloferacaceae can be distinguished from the Halorubraceae and all other archaea by their branching in phylogenetic trees and by the four CSIs listed in Table 1 and by the five CSPs listed in Table 2.

\section{Description of Halorubraceae fam. nov.}

Halorubraceae (Ha.lo.ru.bra.ce'ae. N.L. neut. n. Halorubrum type genus of the family; -aceae ending to denote a family; N.L. neut. pl. n. Halorubraceae the family whose nomenclatural type is the genus Halorubrum).

The family Halorubraceae contains the type genus Halorubrum (Oren et al. 2009) and the genera Halobaculum (Oren et al. 1995), Halohasta (Mou et al. 2012), Halolamina (Cui et al. 2011a), Halonotius (Burns et al. 2010a), Halopenitus (Amoozegar et al. 2012), and Salinigranum (Cui and Zhang 2014). The description of the family is the same as that of the order Haloferacales given by Gupta et al. (2015) with the following modifications: the members of the family Halorubraceae can be distinguished from the Haloferacaceae and all other archaea by their branching in phylogenetic trees and by the four CSPs listed in Table 2. 